\title{
Experimental and Computational Studies of the Structure of CdSe Magic-Size Clusters
}

Igor Dmitruk, ${ }^{a, b, *}$ Rodion V. Belosludov, ${ }^{a}$ Andriy Dmytruk, ${ }^{c, d}$ Yasuto Noda, ${ }^{e, f}$ Yuri Barnakov, ${ }^{c, g}$ Yeon-Su Park, ${ }^{c, h}$ Atsuo Kasuya ${ }^{c, i}$

a Institute for Materials Research, Tohoku University, 2 Chome-1- 1 Katahira, Aoba Ward, Sendai, Miyagi 980-8577, Japan

${ }^{\mathrm{b}}$ National Taras Shevchenko University of Kyiv, Faculty of Physics, 64/13 Volodymyrska str., Kyiv 01601, Ukraine

${ }^{c}$ Center for Interdisciplinary Research,Tohoku University, Aramaki-Aza Aoba 6-3, Sendai 980-8578, Japan

d Institute of Physics, National Academy of Sciences of Ukraine, av. Nauki 46, Kyiv, 03028, Ukraine

${ }^{e}$ Department of Metallurgy, Graduate School of Engineering, Tohoku University, AramakiAza Aoba 6-6-02, Sendai 980-8579, Japan

f JST-CREST, Hon-machi 4-1-8, Kawaguchi, Saitama 332-0012, Japan

${ }^{g}$ Tennessee State University, School of Engineering, Nashville, TN, 3500 John A. Merritt Blvd., Nashville, TN 37209 USA

h Plexense, Inc.\#1-1302, 16-4, Dongbaekjungang-ro 16 beon-gil, Giheung-gu, Yonginsi, Gyeongigo-do 17075, Korea

' NPO Center for Collaborative Interdisciplinary Sciences, Sendai 980-0804, Japan

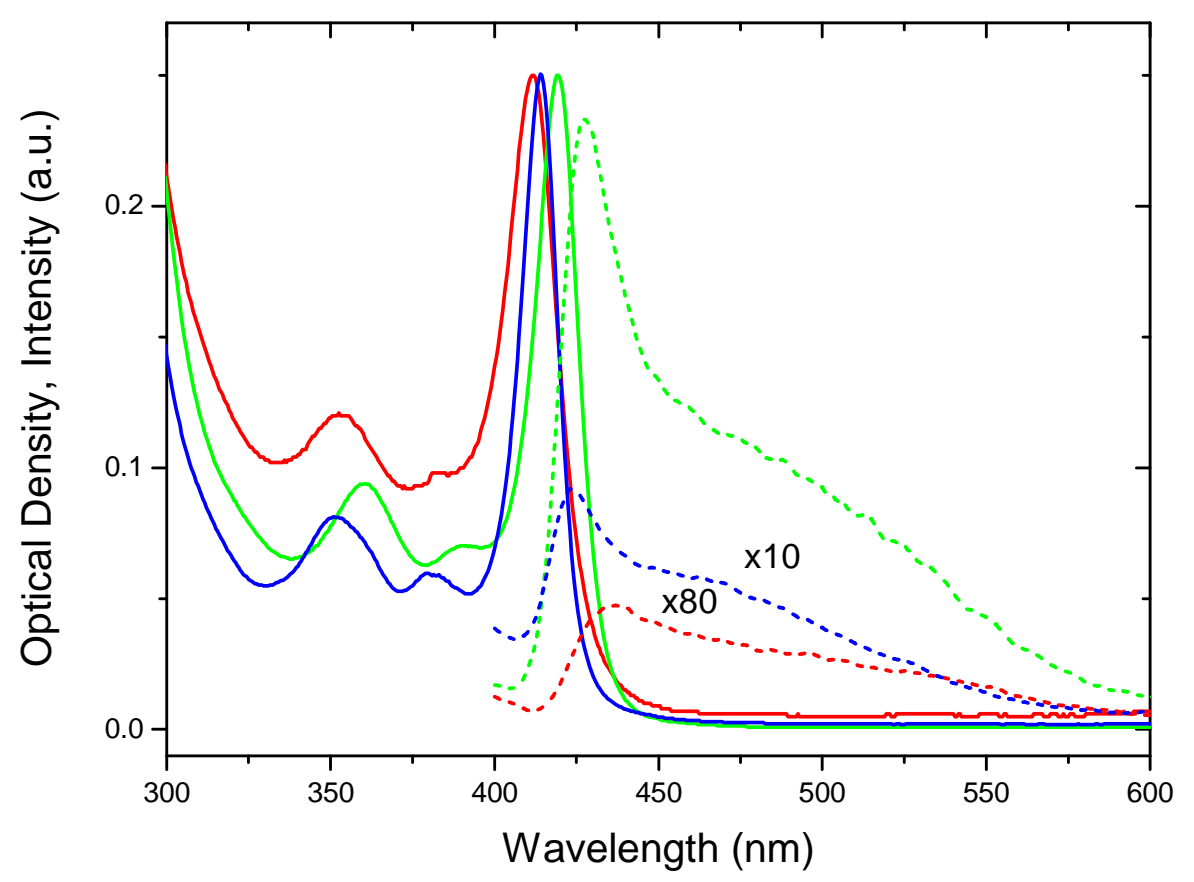

Figure S1. Absorption (solid lines) and photoluminescence (dashed lines) spectra of $(\mathrm{CdSe})_{33}$ and $(\mathrm{CdSe})_{34} \mathrm{NCs}$ covered with different surfactants and dispersed in different solvents: red -CdSe@heptylamine in chlorophorm, green - CdSe@octylamine in toluene, blue-CdSe@cysteine in water. 


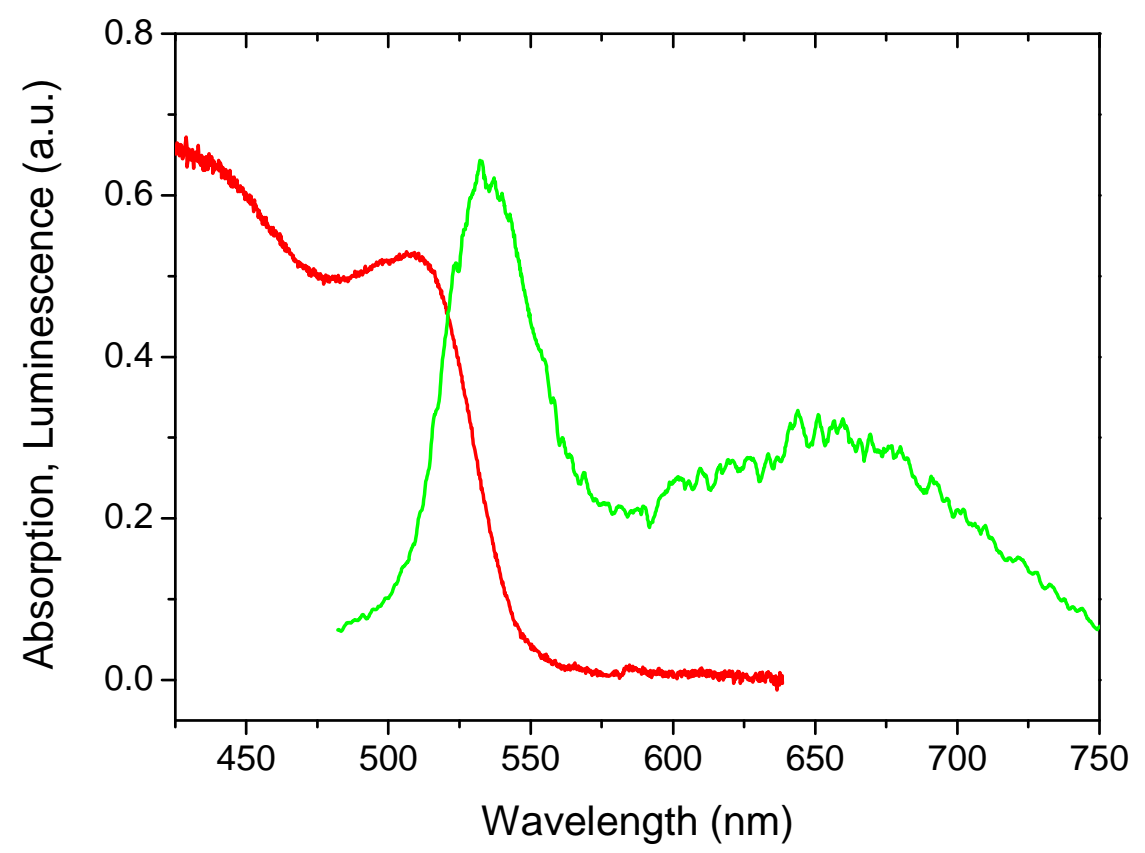

Figure S2. Absorption (red) and photoluminescence (green) spectra of CdSe NPs with $2.2 \mathrm{~nm}$ diameter.

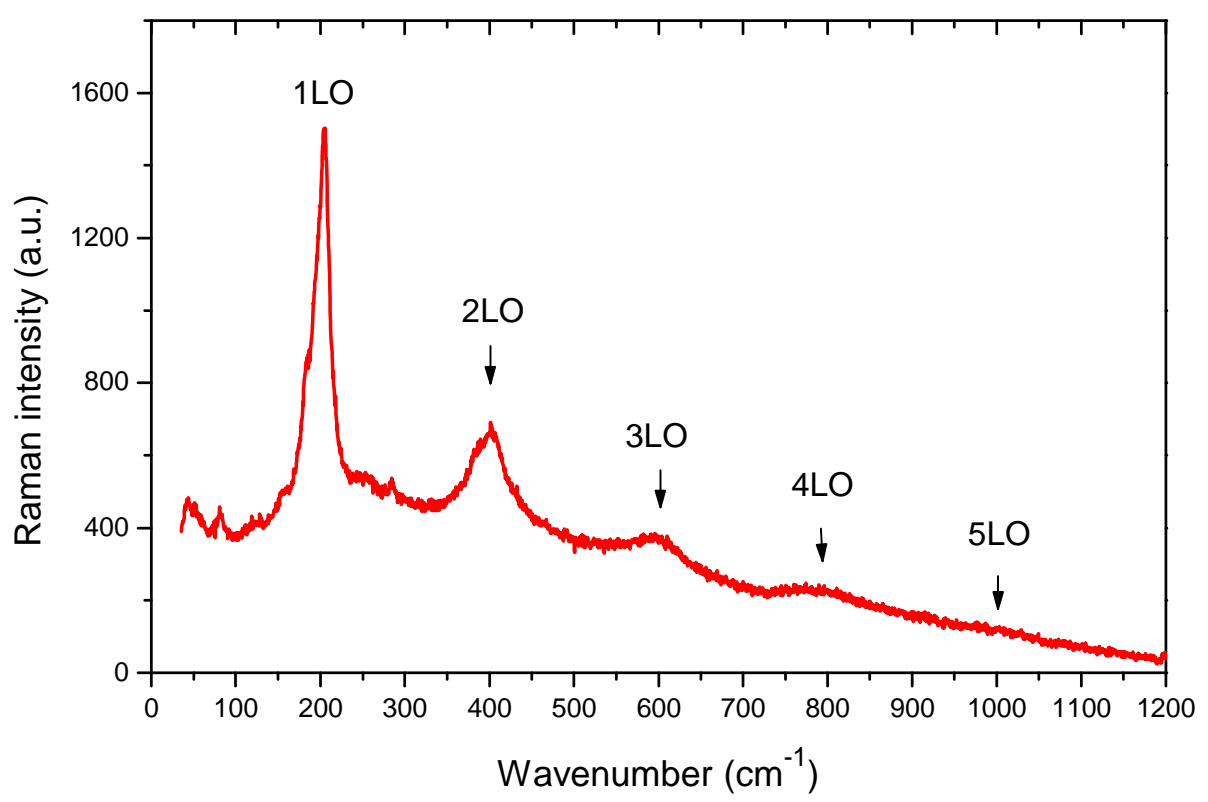

Figure S3. Raman spectrum of CdSe NPs with $2.2 \mathrm{~nm}$ diameter. Excitation wavelength $488 \mathrm{~nm}$, temperature $77 \mathrm{~K}$. 


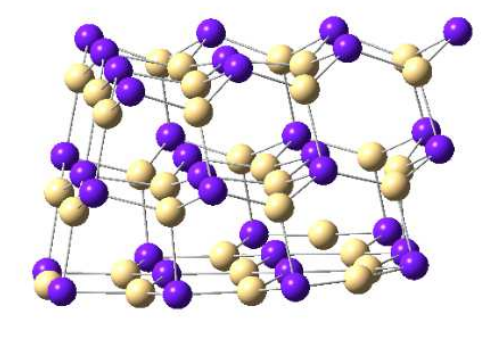

a

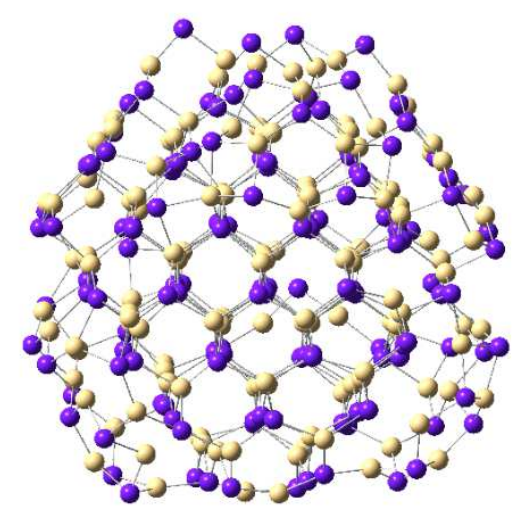

C

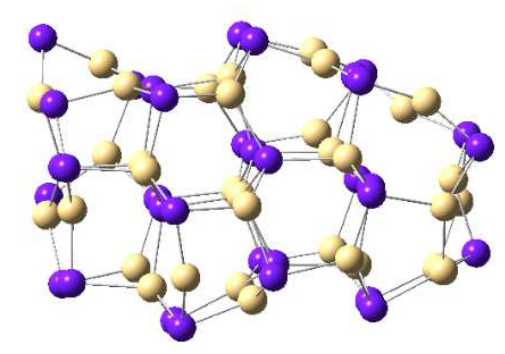

b

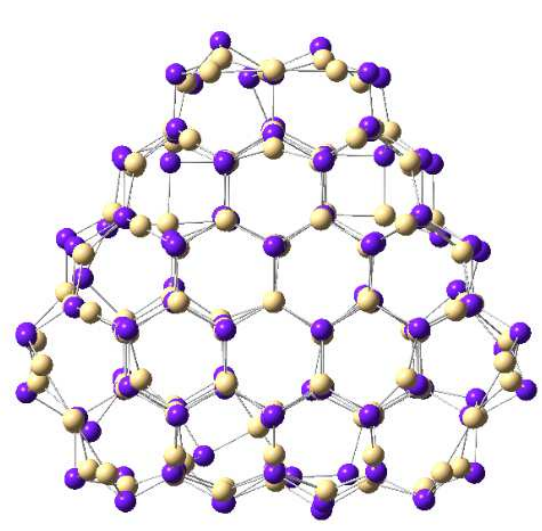

d

Figure S4. Optimized structures of (CdSe) ${ }_{34}$ NPs cut from zinc blende (a) and wurtzite (b) and $2.5 \mathrm{~nm}$ CdSe NPs cut from zinc blende (c) and wurtzite (d) bulk crystal. 


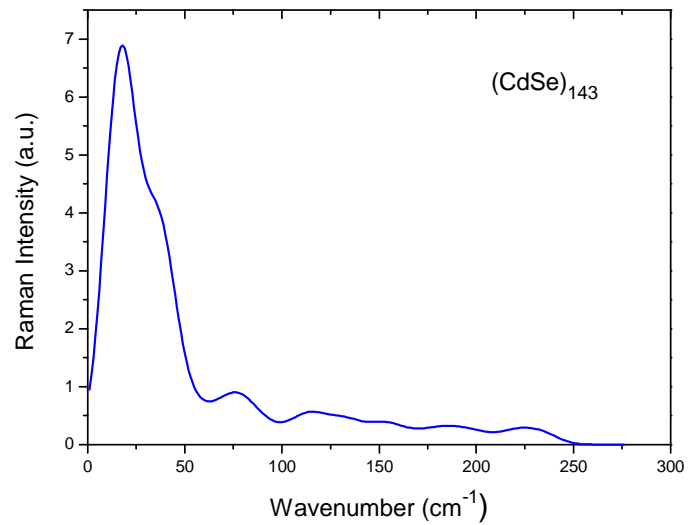

a

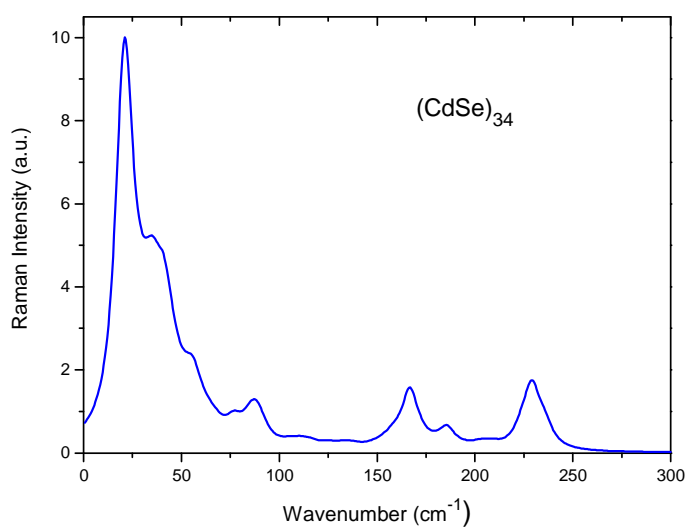

C

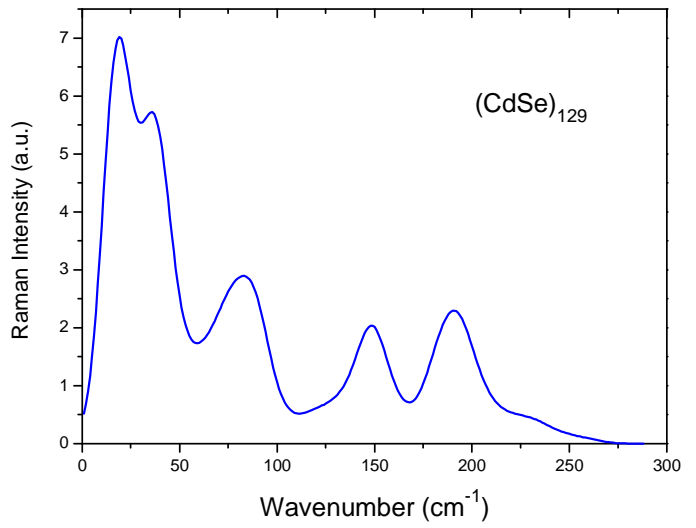

b

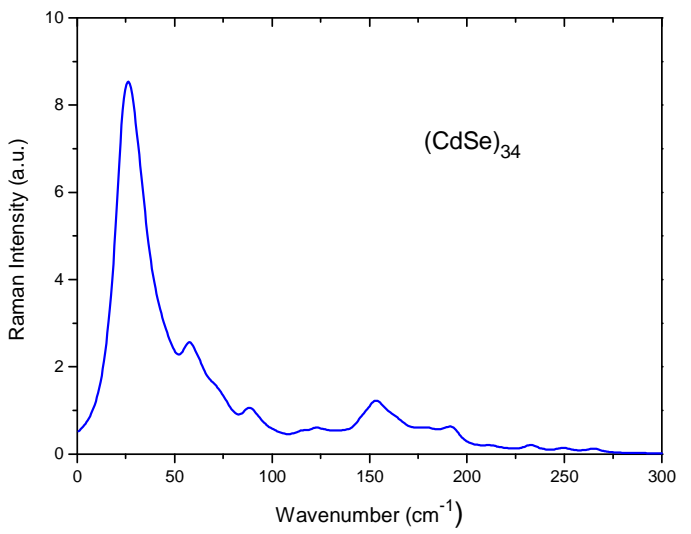

d

Figure S5. Calculated Raman spectra of $2.5 \mathrm{~nm}$ CdSe NPs with zinc blende (a) and wurtzite (b) structure and (CdSe) ${ }_{34}$ NPs with zinc blende (c) and wurtzite (d) structure. 


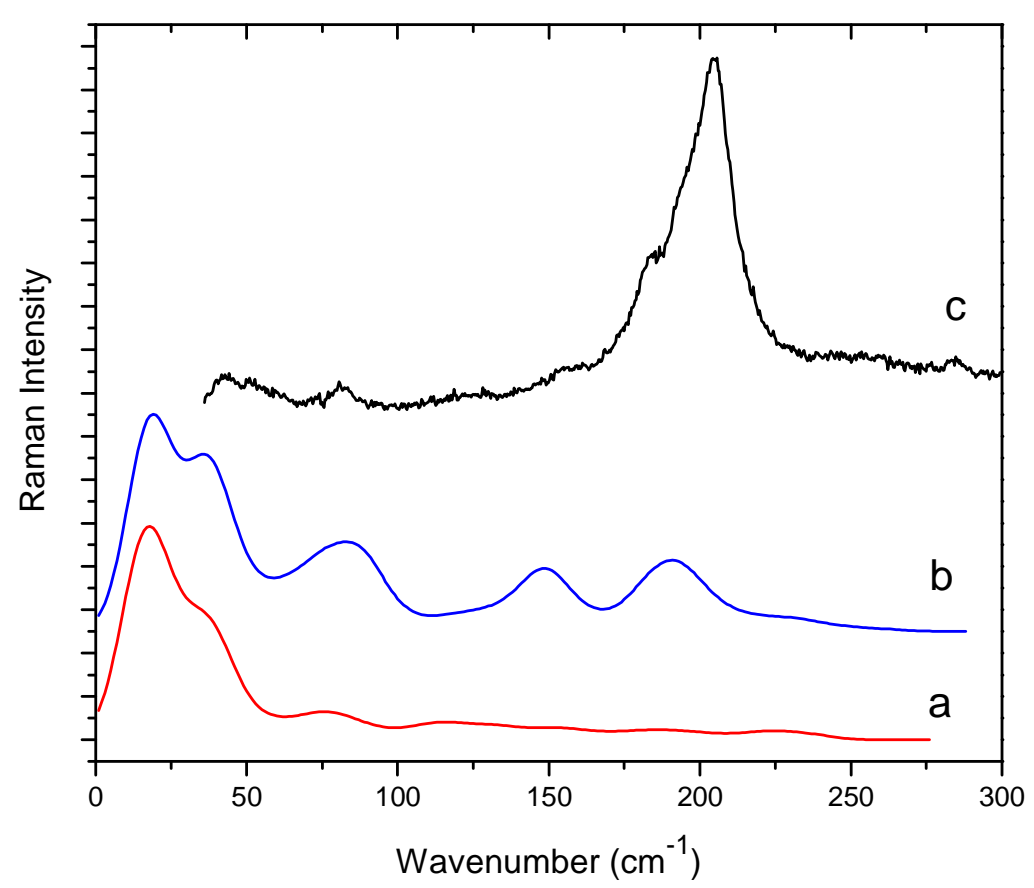

Figure S6. Calculated nonresonant Raman spectra of $2.5 \mathrm{~nm}$ CdSe NPs with zinc blende (a) and wurtzite (b) structure and experimental Raman spectrum of CdSe nanocrystals (c).

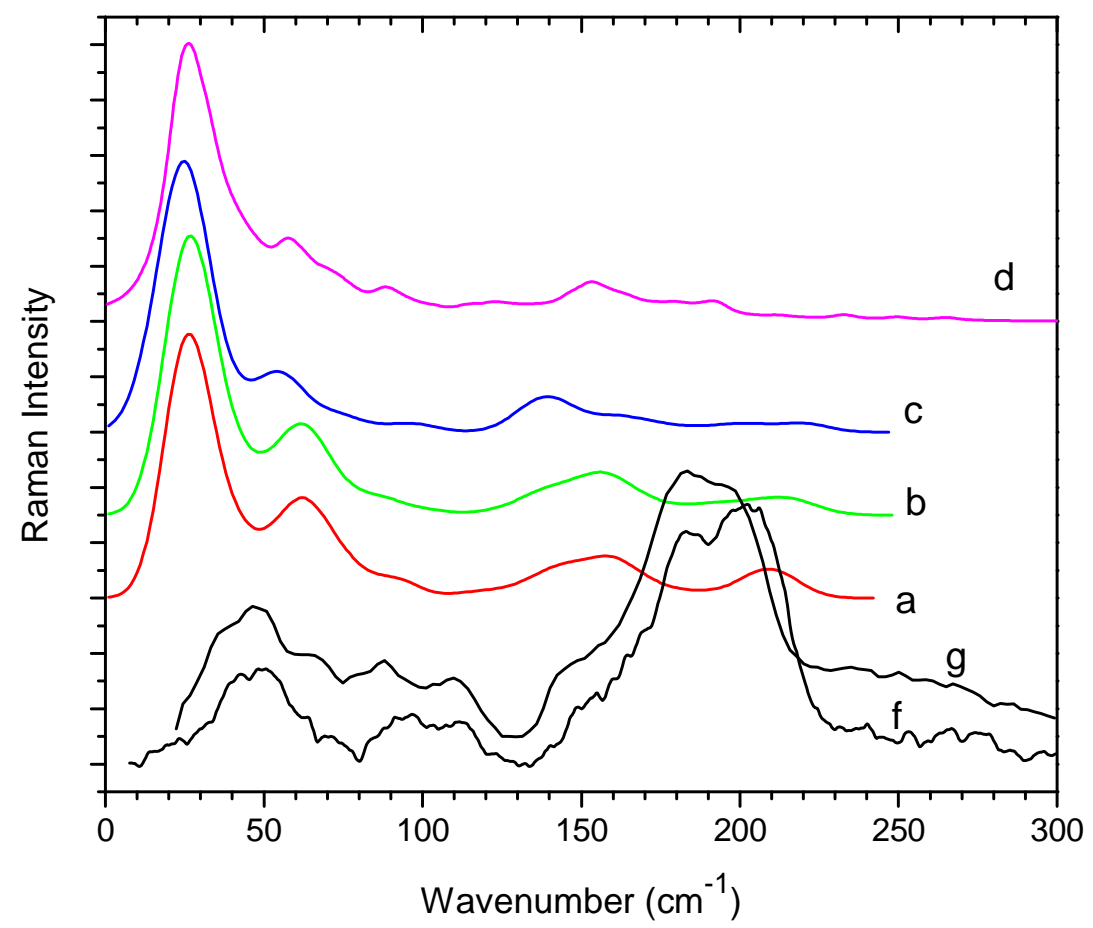

Figure S7. Calculated nonresonant Raman spectra for different structure models of $(\mathrm{CdSe})_{33}$ and $(\mathrm{CdSe})_{34} \mathrm{NCs}:(\mathrm{a})-(\mathrm{CdSe})_{34}(28+6),(\mathrm{b})-(\mathrm{CdSe})_{33}(28+5)$, (c) $(\mathrm{CdSe})_{33}(30+3),(\mathrm{d})-(\mathrm{CdSe})_{34}$ bulk fragment. Black lines present experimental spectra of (f) decylamine and (g) cysteine covered clusters. 


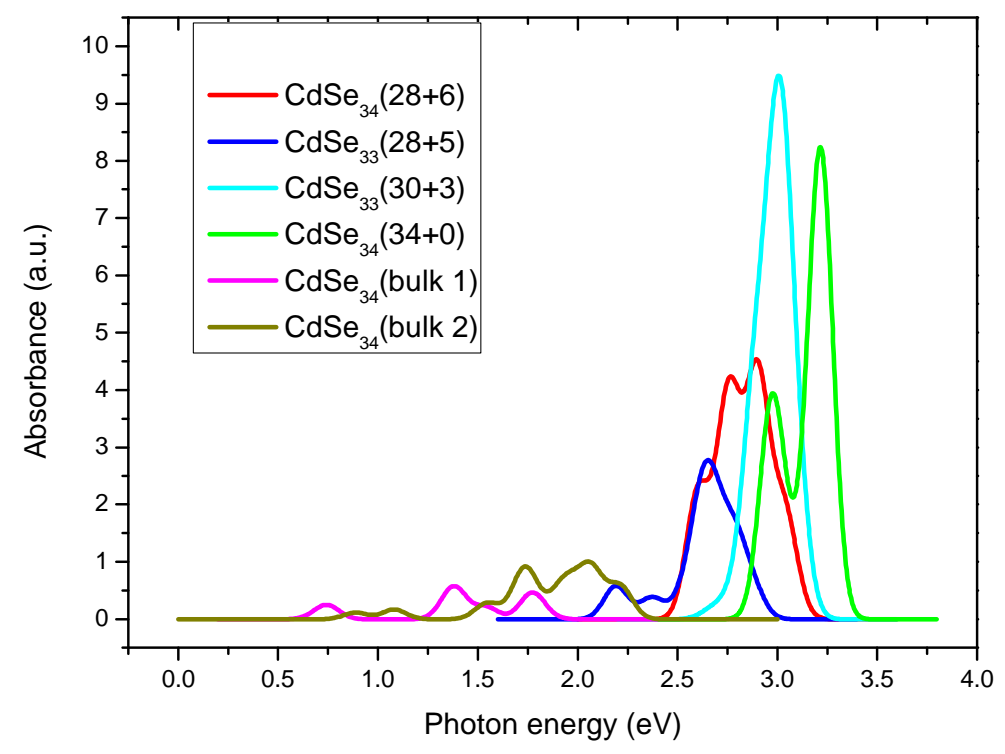

Figure S8. Calculated optical absorption spectra for different structure models of (CdSe $)_{34}$ and $(\mathrm{CdSe})_{33} \mathrm{NCs}$.
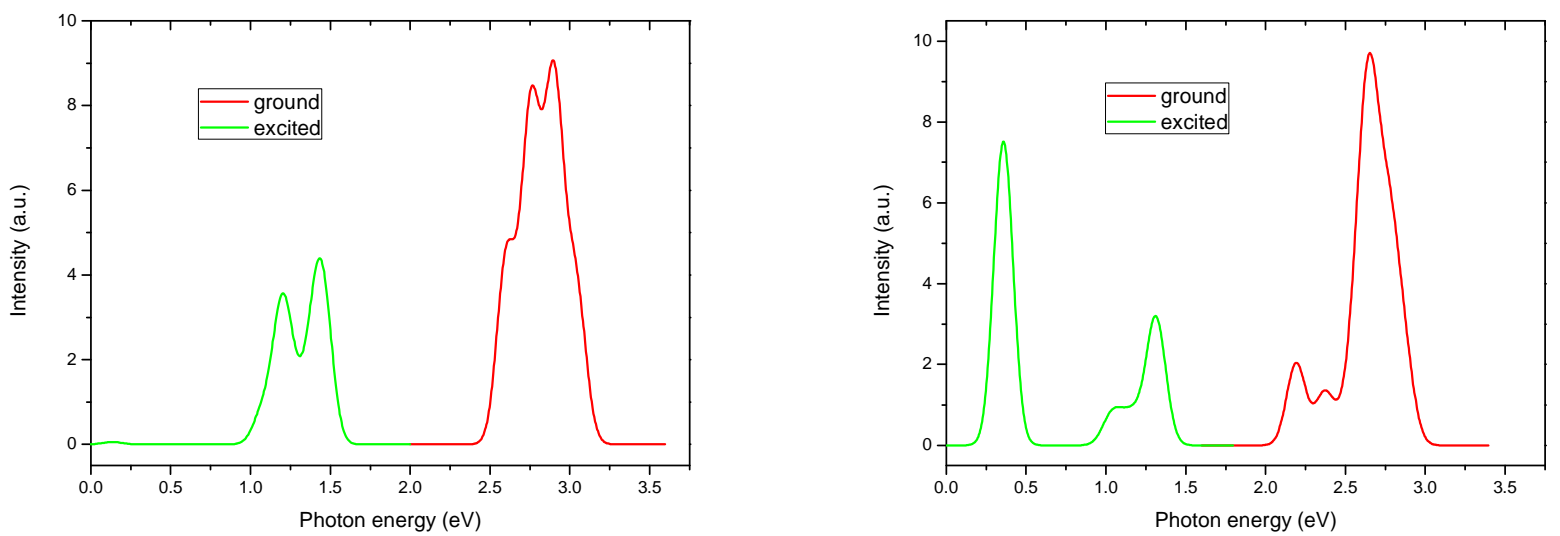

a

b
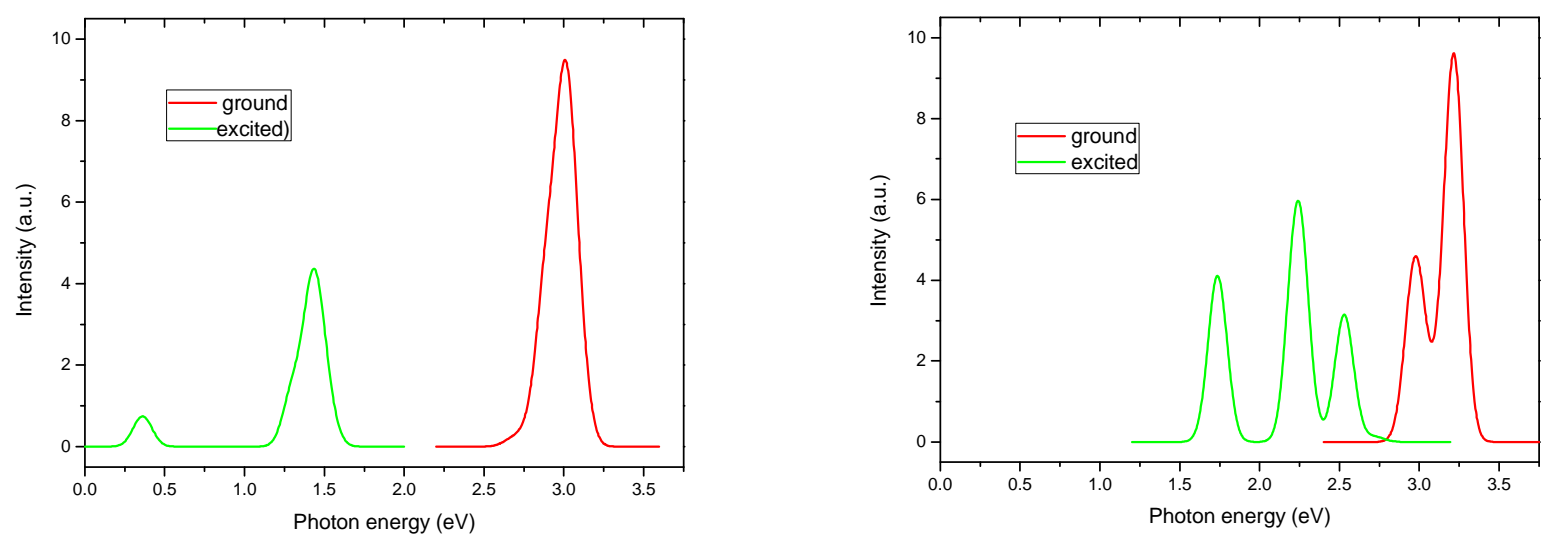

C

$d$

Figure S9. Spectra of electronic transitions calculated for the optimised geometries of the ground and excited states of different structure models of NCs: $(\mathrm{CdSe})_{34}(28+6)(\mathrm{a})$, $(\mathrm{CdSe})_{33}(28+5)(\mathrm{b}),(\mathrm{CdSe})_{33}(30+3)(\mathrm{c}),(\mathrm{CdSe})_{34}(34+0)(\mathrm{d})$. 
Table S1. Coordinates of atoms in optimized structure of $(\mathrm{CdSe})_{34}(28+6) \mathrm{NC}$.

\begin{tabular}{|c|c|c|c|c|c|}
\hline \multirow{2}{*}{$\begin{array}{l}\text { Center } \\
\text { Number }\end{array}$} & \multirow{2}{*}{$\begin{array}{l}\text { Atomic } \\
\text { Number }\end{array}$} & \multirow{2}{*}{\multicolumn{2}{|c|}{$\begin{array}{l}\text { Atomic } \\
\text { Type }\end{array}$}} & \multicolumn{2}{|c|}{ Coordinates (Angstroms) } \\
\hline & & & & $\begin{array}{ll}X & Y\end{array}$ & Z \\
\hline 1 & 48 & 0 & 1.708054 & 1.859504 & 0.927405 \\
\hline 2 & 34 & 0 & 3.715068 & 3.506079 & 1.789650 \\
\hline 3 & 48 & 0 & 1.801550 & 5.443274 & 1.192924 \\
\hline 4 & 34 & 0 & 0.337979 & 6.330384 & 3.172106 \\
\hline 5 & 48 & 0 & -0.200712 & 4.161027 & 4.680254 \\
\hline 6 & 34 & 0 & -2.889897 & 4.450619 & 4.656103 \\
\hline 7 & 48 & 0 & -2.047651 & 5.368374 & 2.243013 \\
\hline 8 & 34 & 0 & -3.373930 & 5.787165 & -0.023561 \\
\hline 9 & 48 & 0 & -4.700557 & 3.435863 & -0.205031 \\
\hline 10 & 34 & 0 & -4.894615 & 1.464227 & 1.787615 \\
\hline 11 & 48 & 0 & -2.464770 & 0.549379 & 0.926393 \\
\hline 12 & 34 & 0 & -2.716250 & 0.442510 & -1.873776 \\
\hline 13 & 48 & 0 & -0.904644 & 2.370315 & -1.621476 \\
\hline 14 & 34 & 0 & -1.567142 & 4.002035 & -3.767254 \\
\hline 15 & 48 & 0 & -1.178310 & 5.633316 & -1.546931 \\
\hline 16 & 34 & 0 & 1.370689 & 6.390856 & -1.271981 \\
\hline 17 & 48 & 0 & 2.905676 & 4.743648 & -2.751320 \\
\hline 18 & 34 & 0 & 5.489368 & 3.861374 & -2.337095 \\
\hline 19 & 48 & 0 & 5.325993 & 2.352805 & -0.202983 \\
\hline 20 & 34 & 0 & 6.698895 & 0.028182 & -0.021928 \\
\hline 21 & 48 & 0 & 5.672485 & -0.911751 & 2.244079 \\
\hline 22 & 34 & 0 & 5.312511 & -3.458999 & 3.172184 \\
\hline 23 & 48 & 0 & 3.702861 & -1.908351 & 4.680587 \\
\hline 24 & 34 & 0 & 1.314258 & -2.320781 & 5.706336 \\
\hline 25 & 48 & 0 & -0.001188 & $3-0.000593$ & 5.516548 \\
\hline 26 & 34 & 0 & -2.668298 & $3 \quad 0.020224$ & 5.705706 \\
\hline 27 & 48 & 0 & -3.487829 & 1.973954 & 4.092708 \\
\hline 28 & 48 & 0 & -3.505181 & -2.254248 & 4.679011 \\
\hline 29 & 34 & 0 & -2.411344 & -4.727932 & 4.655293 \\
\hline 30 & 48 & 0 & 0.032697 & -4.007417 & 4.092859 \\
\hline 31 & 34 & 0 & 1.178522 & -4.971003 & 1.788314 \\
\hline 32 & 48 & 0 & 0.756255 & -2.409322 & 0.926782 \\
\hline 33 & 34 & 0 & 0.975658 & $\begin{array}{ll}3 & -2.573791\end{array}$ & -1.873297 \\
\hline 34 & 48 & 0 & 2.505543 & -0.401222 & -1.621085 \\
\hline 35 & 34 & 0 & 4.250275 & -0.642571 & -3.766648 \\
\hline 36 & 48 & 0 & 1.822966 & -1.715957 & -4.829727 \\
\hline 37 & 34 & 0 & 0.001458 & 0.000633 & -5.768030 \\
\hline 38 & 48 & 0 & -2.396199 & -0.718731 & -4.830618 \\
\hline 39 & 34 & 0 & -2.680646 & -3.358530 & -3.769355 \\
\hline 40 & 48 & 0 & -0.377819 & -4.784113 & -3.993523 \\
\hline 41 & 34 & 0 & 0.600207 & -6.683441 & -2.339173 \\
\hline 42 & 48 & 0 & 2.655752 & -4.887158 & -2.752295 \\
\hline 43 & 34 & 0 & 1.918736 & -4.299031 & -5.336483 \\
\hline 44 & 34 & 0 & 4.849474 & -4.382127 & -1.272281 \\
\hline 45 & 48 & 0 & 3.812837 & -4.282138 & 1.192394 \\
\hline 46 & 48 & 0 & 5.468241 & -1.795827 & -1.546262 \\
\hline 47 & 48 & 0 & -0.625210 & -5.788075 & -0.205261 \\
\hline 48 & 34 & 0 & -3.324871 & -5.815237 & -0.024791 \\
\hline 49 & 48 & 0 & -4.289187 & $7 \quad-3.836934$ & -1.548438 \\
\hline 50 & 34 & 0 & -6.220076 & -2.008473 & -1.274065 \\
\hline 51 & 48 & 0 & -5.560901 & 0.144580 & -2.753175 \\
\hline 52 & 34 & 0 & -6.087746 & 2.822873 & -2.339715 \\
\hline 53 & 48 & 0 & -3.953161 & 2.720878 & -3.993196 \\
\hline 54 & 34 & 0 & -4.680897 & 0.490163 & -5.337559 \\
\hline 55 & 48 & 0 & -5.615747 & $7-1.161484$ & 1.191027 \\
\hline 56 & 34 & 0 & -5.652746 & -2.872518 & 3.170203 \\
\hline 57 & 48 & 0 & -3.626332 & -4.457381 & 2.241730 \\
\hline 58 & 48 & 0 & -1.599772 & -1.968781 & -1.622062 \\
\hline 59 & 48 & 0 & 0.577017 & 2.436569 & -4.829984 \\
\hline 60 & 34 & 0 & 2.766602 & 3.810684 & -5.335735 \\
\hline 61 & 48 & 0 & 4.333987 & 2.064454 & -3.991494 \\
\hline 62 & 34 & 0 & 1.741888 & 2.131667 & -1.872664 \\
\hline 63 & 34 & 0 & 2.749182 & -0.631034 & 1.176820 \\
\hline 64 & 34 & 0 & -1.921237 & -2.065766 & 1.175711 \\
\hline 65 & 34 & 0 & 1.350353 & 2.298723 & 5.706751 \\
\hline 66 & 48 & 0 & 3.452472 & 2.032088 & 4.094162 \\
\hline 67 & 34 & 0 & 5.298236 & 0.275774 & 4.657461 \\
\hline 68 & 34 & 0 & -0.828557 & 2.696289 & 1.176411 \\
\hline
\end{tabular}


Table S2. Coordinates of atoms in optimized structure of $(\mathrm{CdSe})_{33}(28+5) \mathrm{NC}$.

\begin{tabular}{|c|c|c|c|c|c|}
\hline Center & Atomic & & tomic & Coordinates ( & (Angstroms) \\
\hline Number & Number & & Type & $\begin{array}{ll}X & Y\end{array}$ & Z \\
\hline 1 & 48 & 0 & 2.261208 & -2.977113 & 0.591418 \\
\hline 2 & 48 & 0 & 5.078462 & -3.291315 & 1.690601 \\
\hline 3 & 34 & 0 & 6.541442 & -1.257699 & 2.460032 \\
\hline 4 & 48 & 0 & 6.343502 & 0.023346 & 0.086887 \\
\hline 5 & 34 & 0 & 6.656980 & -0.742455 & -2.508337 \\
\hline 6 & 48 & 0 & 5.495168 & 1.709689 & -2.724011 \\
\hline 7 & 34 & 0 & 6.498014 & 2.688328 & -0.415921 \\
\hline 8 & 48 & 0 & 4.307063 & 3.876507 & 0.395927 \\
\hline 9 & 34 & 0 & 3.283983 & 2.754182 & 2.682868 \\
\hline 10 & 48 & 0 & 1.511397 & 1.455633 & 1.079143 \\
\hline 11 & 34 & 0 & 0.499281 & 1.924541 & -1.449031 \\
\hline 12 & 48 & 0 & 1.876316 & 4.090759 & -2.880986 \\
\hline 13 & 34 & 0 & 2.995895 & 5.699009 & -0.982658 \\
\hline 14 & 48 & 0 & 0.933891 & 6.257319 & 0.541793 \\
\hline 15 & 34 & 0 & -1.621643 & 6.799268 & -0.113557 \\
\hline 16 & 48 & 0 & -1.938605 & 4.951082 & -1.963540 \\
\hline 17 & 34 & 0 & -3.847330 & 3.098134 & -1.332573 \\
\hline 18 & 48 & 0 & -1.974331 & 1.365688 & -0.372505 \\
\hline 19 & 34 & 0 & -3.334249 & -0.965344 & -0.555682 \\
\hline 20 & 48 & 0 & -3.861704 & -2.608108 & -2.827420 \\
\hline 21 & 34 & 0 & -3.803337 & -5.079580 & -1.555213 \\
\hline 22 & 48 & 0 & -1.271658 & -5.812314 & -1.578588 \\
\hline 23 & 34 & 0 & 0.647429 & -5.967105 & -3.547605 \\
\hline 24 & 48 & 0 & 0.202784 & -3.400106 & -4.014657 \\
\hline 25 & 34 & 0 & 2.399109 & -1.674242 & -4.039625 \\
\hline 26 & 48 & 0 & 1.316730 & -0.600160 & -1.784392 \\
\hline 27 & 34 & 0 & 0.056140 & -2.858741 & -0.815447 \\
\hline 28 & 48 & 0 & -1.451143 & -1.744452 & 1.175760 \\
\hline 29 & 34 & 0 & -0.854839 & 0.735681 & 2.155494 \\
\hline 30 & 48 & 0 & -2.413521 & 1.935005 & 4.276135 \\
\hline 31 & 34 & 0 & -4.014898 & -0.155930 & 5.007072 \\
\hline 32 & 48 & 0 & -5.625131 & -0.614991 & 2.947069 \\
\hline 33 & 34 & 0 & -6.903346 & 1.172259 & 1.373064 \\
\hline 34 & 48 & 0 & -6.265730 & -0.866820 & -0.348585 \\
\hline 35 & 34 & 0 & -6.435391 & -2.814986 & 1.627763 \\
\hline 36 & 48 & 0 & -4.208552 & -3.882587 & 0.771070 \\
\hline 37 & 34 & 0 & -2.121623 & -3.855299 & 2.786259 \\
\hline 38 & 48 & 0 & 0.107619 & -5.175775 & 2.117224 \\
\hline 39 & 34 & 0 & 0.324960 & -7.046212 & 0.331641 \\
\hline 40 & 48 & 0 & 2.090039 & -5.841682 & -1.321230 \\
\hline 41 & 34 & 0 & 4.375527 & -4.414514 & -0.756174 \\
\hline 42 & 48 & 0 & 4.521188 & -2.232758 & -2.413089 \\
\hline 43 & 34 & 0 & 2.666841 & -4.293121 & 2.982295 \\
\hline 44 & 48 & 0 & 2.069602 & -2.603421 & 4.938699 \\
\hline 45 & 34 & 0 & -0.087850 & -2.158724 & 6.434004 \\
\hline 46 & 48 & 0 & -2.146601 & -1.987178 & 4.805147 \\
\hline 47 & 48 & 0 & 0.907657 & 0.341697 & 6.029856 \\
\hline 48 & 34 & 0 & -0.325490 & 2.639628 & 5.932325 \\
\hline 49 & 48 & 0 & 1.147485 & 3.814655 & 4.083366 \\
\hline 50 & 34 & 0 & 0.510865 & 6.196515 & 3.201224 \\
\hline 51 & 48 & 0 & -1.956638 & 5.588687 & 2.260384 \\
\hline 52 & 34 & 0 & -3.810220 & 4.051464 & 3.283058 \\
\hline 53 & 48 & 0 & -4.955753 & 2.890964 & 1.190777 \\
\hline 54 & 34 & 0 & 3.469068 & -0.448668 & 5.618631 \\
\hline 55 & 48 & 0 & 4.347622 & 0.221570 & 3.212665 \\
\hline 56 & 34 & 0 & -6.151908 & -1.042769 & -3.027764 \\
\hline 57 & 48 & 0 & -4.643812 & 1.129911 & -3.199847 \\
\hline 58 & 34 & 0 & -3.595126 & 1.801296 & -5.540444 \\
\hline 59 & 48 & 0 & -1.917439 & -0.278674 & -5.790014 \\
\hline 60 & 34 & 0 & -2.171634 & -2.741304 & -4.978457 \\
\hline 61 & 34 & 0 & 0.167065 & 1.141065 & -6.768858 \\
\hline 62 & 48 & 0 & 2.192347 & 0.839234 & -5.153578 \\
\hline 63 & 34 & 0 & 3.769060 & 2.804321 & -4.384105 \\
\hline 64 & 48 & 0 & -1.202128 & 3.003667 & -5.352793 \\
\hline 65 & 34 & 0 & -0.343803 & 5.122539 & -4.077415 \\
\hline 66 & 34 & 0 & 3.322439 & -0.515718 & 0.311462 \\
\hline
\end{tabular}


Table S3. Coordinates of atoms in optimized structure of $(\mathrm{CdSe})_{33}(30+3) \mathrm{NC}$.

\begin{tabular}{|c|c|c|c|c|c|}
\hline Center & Atomic & & tomic & Coordinates ( & (Angstroms) \\
\hline Number & Number & & Type & $\begin{array}{ll}X & Y\end{array}$ & Z \\
\hline 1 & 48 & 0 & -1.465508 & 0.490006 & 6.310088 \\
\hline 2 & 48 & 0 & 1.465508 & -0.490006 & 6.310088 \\
\hline 3 & 34 & 0 & 2.336868 & -0.761505 & 3.817791 \\
\hline 4 & 48 & 0 & 3.332633 & 1.494251 & 2.558996 \\
\hline 5 & 34 & 0 & 0.718491 & 2.365280 & 1.349786 \\
\hline 6 & 48 & 0 & -1.884139 & 3.242819 & 2.654984 \\
\hline 7 & 34 & 0 & -2.336868 & 0.761505 & 3.817791 \\
\hline 8 & 48 & 0 & -3.332633 & -1.494251 & 2.558996 \\
\hline 9 & 34 & 0 & -4.975829 & -0.747016 & 0.576188 \\
\hline 10 & 48 & 0 & -4.196772 & 1.561784 & -0.558418 \\
\hline 11 & 34 & 0 & -4.092932 & 3.578150 & 1.181711 \\
\hline 12 & 48 & 0 & -3.348791 & 5.715925 & -0.126130 \\
\hline 13 & 34 & 0 & -1.788977 & 7.738108 & 0.717783 \\
\hline 14 & 48 & 0 & 0.000000 & 6.675283 & 2.371088 \\
\hline 15 & 34 & 0 & -0.835969 & 5.055082 & 4.304615 \\
\hline 16 & 48 & 0 & 1.232515 & 3.579705 & 5.122666 \\
\hline 17 & 34 & 0 & 3.787503 & 3.478295 & 4.302859 \\
\hline 18 & 48 & 0 & 3.876459 & 5.337261 & 2.454387 \\
\hline 19 & 34 & 0 & 5.191047 & 5.366219 & 0.058490 \\
\hline 20 & 48 & 0 & 4.599709 & 2.972649 & -0.778349 \\
\hline 21 & 34 & 0 & 2.925956 & 2.699431 & -2.907072 \\
\hline 22 & 48 & 0 & 2.631072 & 0.153612 & -4.127795 \\
\hline 23 & 34 & 0 & 0.000000 & 0.000000 & -2.808178 \\
\hline 24 & 48 & 0 & -2.631072 & -0.153612 & -4.127795 \\
\hline 25 & 34 & 0 & -2.925956 & -2.699431 & -2.907072 \\
\hline 26 & 48 & 0 & -4.599709 & -2.972649 & -0.778349 \\
\hline 27 & 34 & 0 & -5.191047 & -5.366219 & 0.058490 \\
\hline 28 & 48 & 0 & -3.876459 & -5.337261 & 2.454387 \\
\hline 29 & 34 & 0 & -3.787503 & -3.478295 & 4.302859 \\
\hline 30 & 48 & 0 & -1.232515 & -3.579705 & 5.122666 \\
\hline 31 & 34 & 0 & 0.835969 & -5.055082 & 4.304615 \\
\hline 32 & 48 & 0 & 1.884139 & -3.242819 & 2.654984 \\
\hline 33 & 34 & 0 & 4.092932 & -3.578150 & 1.181711 \\
\hline 34 & 48 & 0 & 4.196772 & -1.561784 & -0.558418 \\
\hline 35 & 34 & 0 & 4.975829 & 0.747016 & 0.576188 \\
\hline 36 & 34 & 0 & 4.170175 & -1.815283 & -3.201455 \\
\hline 37 & 48 & 0 & 2.655990 & -3.926521 & -3.774535 \\
\hline 38 & 34 & 0 & 3.177338 & -6.295311 & -2.736496 \\
\hline 39 & 48 & 0 & 3.348791 & -5.715925 & -0.126130 \\
\hline 40 & 34 & 0 & 1.788977 & -7.738108 & 0.717783 \\
\hline 41 & 48 & 0 & 0.000000 & -6.675283 & 2.371088 \\
\hline 42 & 34 & 0 & -2.468746 & -7.566014 & 2.011995 \\
\hline 43 & 48 & 0 & -2.965653 & -6.777540 & -0.504265 \\
\hline 44 & 34 & 0 & -1.590828 & -7.118256 & -2.744739 \\
\hline 45 & 48 & 0 & -1.335893 & -4.700745 & -3.821117 \\
\hline 46 & 34 & 0 & 0.609868 & -4.141651 & -5.519834 \\
\hline 47 & 48 & 0 & 0.189483 & -1.667896 & -6.336234 \\
\hline 48 & 34 & 0 & 2.058205 & 0.253729 & -6.711047 \\
\hline 49 & 48 & 0 & -0.189483 & 1.667896 & -6.336234 \\
\hline 50 & 34 & 0 & -2.058205 & -0.253729 & -6.711047 \\
\hline 51 & 34 & 0 & -0.609868 & 4.141651 & -5.519834 \\
\hline 52 & 48 & 0 & 1.335893 & 4.700745 & -3.821117 \\
\hline 53 & 34 & 0 & 1.590828 & 7.118256 & -2.744739 \\
\hline 54 & 48 & 0 & -0.862726 & 7.263948 & -1.743813 \\
\hline 55 & 34 & 0 & -3.177338 & 6.295311 & -2.736496 \\
\hline 56 & 48 & 0 & -2.655990 & 3.926521 & -3.774535 \\
\hline 57 & 34 & 0 & -4.170175 & 1.815283 & -3.201455 \\
\hline 58 & 48 & 0 & 2.965653 & 6.777540 & -0.504265 \\
\hline 59 & 34 & 0 & 2.468746 & 7.566014 & 2.011995 \\
\hline 60 & 48 & 0 & 0.862726 & -7.263948 & -1.743813 \\
\hline 61 & 34 & 0 & -0.650927 & -1.933439 & 7.157464 \\
\hline 62 & 48 & 0 & -1.052194 & -1.768820 & -1.157292 \\
\hline 63 & 34 & 0 & -0.718491 & -2.365280 & 1.349786 \\
\hline 64 & 48 & 0 & 0.000000 & 0.000000 & 2.551712 \\
\hline 65 & 48 & 0 & 1.052194 & 1.768820 & -1.157292 \\
\hline 66 & 34 & 0 & 0.650927 & 1.933439 & 7.157464 \\
\hline
\end{tabular}

